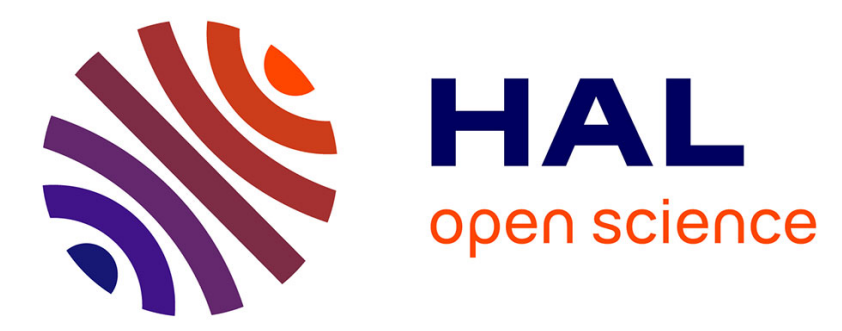

\title{
MODEL-DRIVEN PARAMETERIZATION OF FETAL CORTICAL SURFACES
}

Guillaume Auzias, François de Guio, Antonietta Pepe, François Rousseau, Jean-François Mangin, Nadine Girard, Julien Lefèvre, Olivier Coulon

\section{- To cite this version:}

Guillaume Auzias, François de Guio, Antonietta Pepe, François Rousseau, Jean-François Mangin, et al.. MODEL-DRIVEN PARAMETERIZATION OF FETAL CORTICAL SURFACES. 2015. hal01114993

\section{HAL Id: hal-01114993 \\ https://hal.science/hal-01114993}

Preprint submitted on 10 Feb 2015

HAL is a multi-disciplinary open access archive for the deposit and dissemination of scientific research documents, whether they are published or not. The documents may come from teaching and research institutions in France or abroad, or from public or private research centers.
L'archive ouverte pluridisciplinaire HAL, est destinée au dépôt et à la diffusion de documents scientifiques de niveau recherche, publiés ou non, émanant des établissements d'enseignement et de recherche français ou étrangers, des laboratoires publics ou privés. 


\title{
MODEL-DRIVEN PARAMETERIZATION OF FETAL CORTICAL SURFACES
}

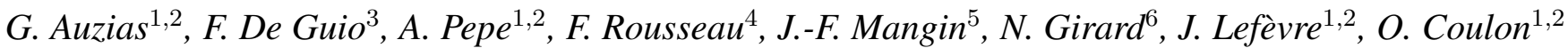 \\ ${ }^{1}$ Institut de Neurosciences de la Timone UMR 7289, Aix Marseille Université , CNRS, Marseille, France \\ ${ }^{2}$ Aix Marseille Université, CNRS, Laboratoire LSIS, UMR 7296, Marseille, France \\ ${ }^{3}$ Univ Paris Diderot, Sorbonne Paris Cité, UMR-S 1161 INSERM, Paris, France \\ ${ }^{4}$ ICube, Université de Strasbourg/CNRS, UMR 7357, Illkirch, France \\ ${ }^{5}$ NeuroSpin, CEA, Gif/Yvette, France \\ ${ }^{6}$ Aix Marseille Université, CNRS, CRMBM UMR 7339, Marseille, France
}

\begin{abstract}
Surface-based analysis is a tool of choice to study the anatomy and function of the cortex in adult and children brains. Common surface registration approaches are not adaptable to fetal data since data-driven mapping techniques are limited by the lack of structural features across all gestational ages. In this work, we adapt the HIP-HOP modeldriven cortical surface parameterization method to the specific problem of defining correspondences across fetuses with different gestational ages. We demonstrate the validity of our approach by quantifying the curvature evolution during development. Our findings are highly consistent with previous studies. This work is the first demonstration of the feasibility of applying surface-based mapping and analysis to fetal data.
\end{abstract}

Index Terms - MRI, brain, surface, mapping

\section{INTRODUCTION}

Recent advances in motion-corrected 3D MRI reconstruction and segmentation of developing tissues in the fetal brain enables quantitative analysis at fine spatial resolution $[1,2,3]$. Surface-based analysis is a tool of choice to study the anatomy and function of the cortex in adult and children brains [4], but its application to fetal data is limited because current surface registration techniques are not appropriate [2]. Indeed, the major variations in curvature magnitude occurring during antenatal development limit the use of surfacic alignment methods driven by curvature such as [5]. Methods based on sulcal landmarks such as in [6] could be applied to define a mapping between cortical surfaces from several individuals at a given gestational age (GA) as the folding pattern evolution during antenatal life has been described, with notable reproducibility across individuals [2]. The drastic changes in the folding with GA however results in variations in the topology and number of sulcal landmarks that interfere with the basic

This work has been supported by the Agence Nationale de la Recherche (ANR MoDeGy, ANR-12-JS03-001-01). one-to-one assumption previous approaches rely on. As a whole, data-driven surface registration techniques are not appropriate due to the lack of consistent structural features such as the primary sulci across all GAs, especially for young fetuses. In this work, we present an adaptation of the HIP-HOP model-driven cortical surface parameterization method [7] to the specific problem of defining correspondences across fetuses with different GA. Using structural information from in utero MRI and defining a common model across GAs, we perform morphometric analysis of early folding during cortical plate surface development in the normal fetal brain between 21 to 34 weeks of gestation. Our results are highly consistent with previous studies based on volumetric registration technique $[2,3]$, which validate the implementation of our model-driven approach. The surface-based analysis framework we propose will be of key interest for probing neurodevelopmental disorders in the future.

\section{MATERIAL AND METHODS}

\subsection{Data Acquisition and Preprocessing}

We considered 15 fetuses with no apparent anatomical abnormalities and whose GAs range from 21 to 34 weeks. Clinical MR imaging was performed on a $1.5 \mathrm{~T}$ scanner. All mothers gave informed consent for the study. For each subject, three stacks of HASTE sequence T2 $\mathrm{w}$ slice images (resolution $0.742 \times 0.742 \times 3 \mathrm{~mm}^{3}$ ) were obtained in the approximately axial, sagittal and coronal planes with respect to the fetal brain. High-resolution isotropic images $(0.75 \times 0.75 \times 0.75$ $\mathrm{mm}^{3}$ ) were reconstructed from the three stacks using a slice intersection motion correction technique [1]. A dedicated semi-automatic procedure was used for the segmentation of the developing cortex and white matter. A smooth trianglebased mesh of the gray and white matter interface was computed and corrected manually when deemed necessary as described [8]. The surface mean curvature was estimated from the mesh local geometry with positive curvatures correspond- 
ing to the gyri (convex regions), and negative curvatures corresponding to the bottom of folds (concave regions). Lines following the fundus of major sulci were manually traced based on geodesics constrained by curvature maps using the surfPaint interactive tool [9] that is available in the BrainVISA $^{1}$ free software platform.

\subsection{Model-driven Parameterization}

We presented in [7] a method for defining a parameterization of the cortical surface constrained by a model of cortical organization denoted as HIP-HOP. The main idea of the model originally presented in [10] is the spatial stability of deeply buried subparts of sulci, corresponding to the first folding location during antenatal life. In this model, the major sulci serve as landmarks that are organized in two sets of orthogonal constraints (longitudes/latitudes) relatively to two poles defined as the insula and the cingular pole. As detailed in $[10,7]$, this model corresponds to a coordinate system in which the folds that are part of the model always have the same orientation and coordinate. Left panel of Fig.1 illustrates the model-based parameterization scheme.

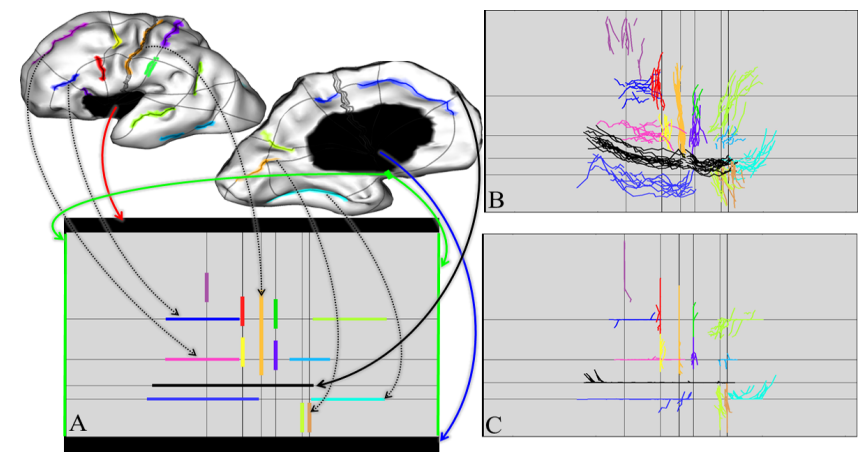

Fig. 1. Scheme of the model-driven parameterization procedure. Each individual surface is mapped onto a rectangular domain corresponding to the model (A). The arrows indicate the expected correspondences resulting from the mapping for some of the constraints. (B) shows the superposition of the 15 individuals after unconstrained mapping. (C) The dispersion of sulci around the axes is reduced by the constrained mapping.

Formally, HIP-HOP consists in defining an harmonic mapping of each hemisphere surface to a rectangular planar domain that integrates a representation of the model (Fig.1.A). Let us denote $S_{T} \in \mathbf{R}^{3}$ the cortical mesh. The parameterization of $S_{T}$ consists in defining a rectangular domain $\Omega \subset \mathbf{R}^{2}$ and a continuous mapping $h: S_{T} \rightarrow \Omega$ that is linear on each triangle in $S_{T}$. Such a mapping is uniquely determined by the images $u=\left(u_{1}, u_{2}\right)=h(v)=$ $\left(h_{1}\left(v_{1}, v_{2}, v_{3}\right), h_{2}\left(v_{1}, v_{2}, v_{3}\right)\right) \in \mathbf{R}^{2}$. As illustrated on

\footnotetext{
${ }^{1}$ http://brainvisa.info
}

fig.1.B and $\mathrm{C}$, we decompose the parameterization of the cortical surface into two steps.

Unconstrained Mapping Onto a Rectangle: We first define a mapping $f: S_{T} \rightarrow \Omega$ from the surface onto a $2 \mathrm{D}$ rectangle by minimizing the Dirichlet energy: $E_{D}(f)=$ $1 / 2 \int_{S_{T}}\left\|\nabla_{S_{T}} f\right\|^{2}$. The resulting mapping is harmonic and thus preserves the angles between the original surface and the flattened rectangle. As detailed in [7], the location and orientation of sulci in the rectangle follow the organization on the original surface.

Aligning Sulci Onto Corresponding Axes of the Model: We then define two sets of constraints corresponding to the sulcal lines specified in the anatomical model as latitudes and longitudes respectively. The constrained mapping $g: \Omega \rightarrow \Omega$ that aligns the sulci onto the model while minimizing angle distortion is defined as the optimum of the following energy: $E(g)=\frac{1}{2} \int_{\Omega}\left\|\nabla_{S_{T}} g\right\|^{2}+\frac{\lambda}{2} \int_{\Omega}\|g(u)-p(g(u))\|^{2} d u$, where $p(g(u))$ is the projection of a point onto the corresponding axis. The parameterization mapping $h$ is then defined as the composition of $f$ and $g: h=g \circ f$.

As opposed to other landmark-based registration methods, we do not match folds between individuals but instead optimize the fit between cortical sulci and specific iso-coordinate axis in the model. This strategy enables the alignment of sulci across individuals without requiring that landmarks have the same topology: a multiple parts sulcus can be aligned onto an axis as well as a continuous one. HIP-HOP thus overcomes the issue of folding magnitude variations across individuals and GA, and is particularly well-suited for fetal data analysis. In order to further adapt this technique to fetal data, we need to 1) define a specific model that is different from our previous work on adult brains $[10,7]$ and 2) design a procedure that accommodates variations in the number of landmarks across individuals and GAs, including younger fetuses with no identifiable sulcus.

\subsection{Data-driven Model of Fetal Brain}

The coordinate values of the axes of the model (longitude or latitude) are defined for a group of subjects after the unconstrained mapping in the rectangular domain with minimal geometric distortion (Fig.1.B, [7]). Each sulcus that can be identified in at least two individuals is included in the model. For each axis of the model, we compute the barycenter of the points associated to the corresponding sulcal landmark across the subjects of the group. The longitude (resp. latitude) value of the axis then corresponds to the first (resp. second) coordinate of the barycenter. Note that some sulci cannot be identified in every individuals. Therefore, the model is computed from the maximal sulcal information available across the group of subjects, and some sulci are more present -and thus have more weight in the model- than others. 


\subsection{Fetuses-specific Supplementary Anatomical Con- straints}

For younger individuals ( $\sim 21$ week GA), many sulci cannot be identified [2]. In this case, the parameterization cannot be driven by sulcal landmarks, but the delineation of the insular and cingular poles is always possible. We suggest to include in the model two other types of constraints that can be identified in every brain. First, we consider the line of maximal curvature that delimitates the medial face from the external face of the cortical surface. It can be seen on Fig. 1 and 2 in black on individual surfaces, with corresponding straight black lines on the rectangular representations on Fig.1.A,B and C. Second, as shown in [11], the two extrema of the second eigenfunction of the Laplace-beltrami operator are the two points the farthest to each other on the surface. In our case the most anterior and posterior points on the cortex. The barycenter of these two points in the rectangular domain is computed for each subject. When the central suclus cannot be identified, this barycenter is used instead: it is matched onto the longitude coordinate corresponding to the average location across our group of subjects for this particular landmark. For such young fetuses, the mapping onto the model is only constrained by the insular and cingular poles and these two specific constraints. When additional sulcal landmarks can be traced for an individual, they serve as additional constraints in the definition of the model. Even in the case of a small number of constraints, the harmonic regularization term in $E(g)$ effectively provides a smooth and efficient HIP-HOP mapping, as presented in results, section 3.1.

\subsection{Experiments: Surface-based Analysis of Cortical Folding With Age}

In order to provide evidence for the validity of inter-individuals correspondences resulting from our approach, we reproduced recent results from [2] where a volumetric registration technique was used. We quantified the evolution of the cortical surface during early cortical folding based on local curvature measurements. The cortical surface of the largest brain was used as a spatial support to project individual maps of mean curvature, using the inter-subject correspondences provided by our method (Fig.3.A). A group analysis of these maps was performed using SurfStat ${ }^{2}$, a toolbox dedicated to surface-based statistical analysis of neuroimaging data. We used linear regression models to assess the effect of age on local curvature at each vertex of the surface. The resulting regression coefficients provided an estimate of the local rate of folding of the cortical surface. Findings from our surfacebased analysis were controlled for multiple comparison using random field theory as implemented in SurfSat. Specifically, significances were thresholded at $p<0.05$ after correction for family-wise error rate at cluster level.

\footnotetext{
${ }^{2}$ http://www.math.mcgill.ca/keith/surfstat
}

\section{RESULTS AND DISCUSSION}

\subsection{Interindividual Correspondences}

Fig.2 shows the axes of the model mapped onto the cortical surface of 4 individuals spanning the age range of the population. Note how the location of iso-coordinate axes is reproducible across individuals while the folding magnitude increases with age. This shows the accuracy of the correspondences across individuals that results from the mapping of each surface onto a common model with HIP-HOP.

For the younger individual on the left, no sulcal landmark could be identified, and the location of the axes of the model are obtained from the alignment of only the poles boundary and specific landmarks as detailed in section 2.4. The good fit of the parameterization even for this subject illustrates the interest of our model-driven approach for fetal data analysis.

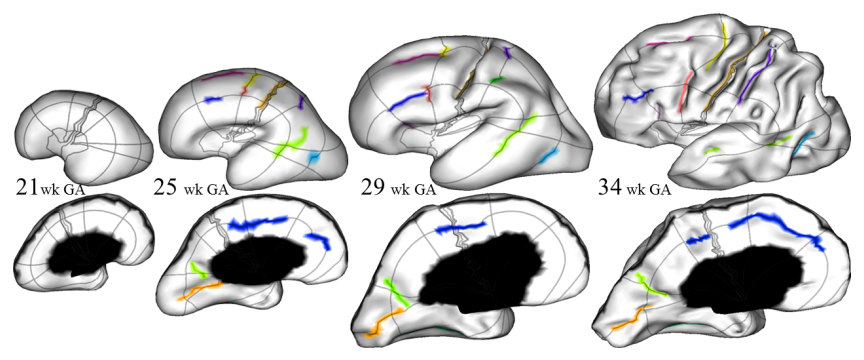

Fig. 2. The axes of the model mapped onto the cortical surface of 4 individuals spanning the age range of the population. These axes have the same coordinate across subjects and illustrate the accuracy of the inter-individual correspondences.

\subsection{Folding Evolution Quantified With Curvature}

Fig.3.A shows the average of individual mean curvature maps across all 15 subjects projected on the largest surface (section 2.5). The sharpness of local variations of this average curvature map shows that the folds have been aligned consistently across subjects.

We detected changes in brain folding that were significantly correlated with age: on fig.3.B, regions in blue (resp. red) indicate significant increase in concavity (resp. convexity), i.e. negative curvature (resp. positive curvature). An increase of concavity was detected in two regions of the insula (INS). A nearby significant increase in convexity occurred along the superior temporal gyrus (STG). The emergence of central sulcus (CS) is shown by a significant increase of concavity in its superior and inferior parts, together with increases of concavity on the precentral gyrus (PG). In the frontal lobe, the anterior and the posterior parts of the superior frontal sulcus (SFS) show significant increase of concavity. Regions directly anterior to the future precentral gyrus also increase in concavity. On the medial face, the precuneus $(\mathrm{PCu})$, the parieto-occipital sulcus $(\mathrm{PoS})$ and the 
calcarine sulcus (Cal) underwent an increase in curvature. In the medial temporal lobe, the emergence of the collateral sulcus (Coll) is reflected by a significant increase in concavity. For two of these regions, local mean curvature was plotted as a function of GA (fig.3.C). The resulting graphs illustrate the strong linear relationship between local curvature and age in convex (left) and concave (right) region. Despite the relatively small sample size, our results are highly consistent with previous findings on curvature evolution using volumetric registration technique [2].

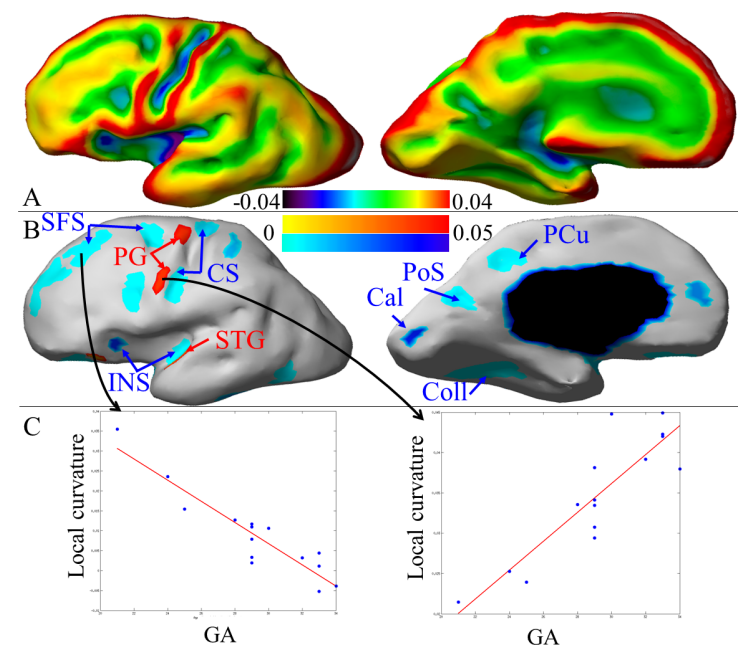

Fig. 3. (A) Average of mean curvature maps. (B) Clusters of significant increase in positive (red) and negative (blue) local curvature, after cluster-level correction for multiple comparisons. (C) Local mean curvature as a function of GA in convex (left) and concave (right) regions.

\section{CONCLUSION}

Data-driven surface registration techniques are not effective for fetal data due to the lack of consistent structural features across all gestational ages. Using model-driven strategy is of key interest in this context and HIP-HOP is the only available method. We detailed the definition of a dedicated model and corresponding tunning of the mapping technique. The application of this approach to the analysis of curvature evolution during development resulted in findings that are highly consistent with previous study. This work is the first demonstration of the feasibility of applying surface-based mapping and analysis to fetal data. Such quantitative regional information are of key interest for probing in utero neurodevelopmental disorders in the future.

\section{REFERENCES}

[1] F. Rousseau, O. Glenn, B. Iordanova, C. RodriguezCarranza, D. Vigneron, J. Barkovich, and C. Studholme,
"Registration-based approach for reconstruction of high-resolution in utero fetal MR brain images.," Academic radiology, vol. 13, no. 9, pp. 1072-81, 2006.

[2] P. Habas, J. Scott, A. Roosta, V. Rajagopalan, K. Kim, F. Rousseau, J. Barkovich, O. Glenn, and C. Studholme, "Early folding patterns and asymmetries of the normal human brain detected from in utero MRI.," Cerebral cortex, vol. 22, no. 1, pp. 13-25, 2012.

[3] R. Wright, V. Kyriakopoulou, C. Ledig, M. Rutherford, J. Hajnal, D. Rueckert, and P. Aljabar, "Automatic quantification of normal cortical folding patterns from fetal brain MRI.," NeuroImage, vol. 91, pp. 21-32, 2014.

[4] D. Van Essen, H. Drury, S. Joshi, and M. Miller, "Functional and structural mapping of human cerebral cortex: solutions are in the surfaces.," PNAS, vol. 95, no. 3, pp. 788-95, 1998.

[5] B. Fischl, A. Van der Kouwe, C. Destrieux, E. Halgren, F. Ségonne, D. Salat, E. Busa, L. Seidman, J. Goldstein, D. Kennedy, V. Caviness, N. Makris, B. Rosen, and A. Dale, "Automatically parcellating the human cerebral cortex," Cerebral Cortex, vol. 14, no. 1, pp. 11, 2004.

[6] J. Hill, D. Dierker, J. Neil, T. Inder, A. Knutsen, J. Harwell, T. Coalson, and D. Van Essen, "A surfacebased analysis of hemispheric asymmetries and folding of cerebral cortex in term-born human infants.," J. Neuroscience, vol. 30, no. 6, pp. 2268-76, 2010.

[7] G. Auzias, J. Lefèvre, A. Le Troter, C. Fischer, M. Perrot, J. Régis, and O. Coulon, "Model-driven Harmonic Parameterization of the Cortical Surface: HIP-HOP," IEEE Trans. Med. Imag., vol. 32, no. 5, pp. 873-887, 2013.

[8] J. Dubois, M. Benders, A. Cachia, F. Lazeyras, R. HaVinh Leuchter, S.V. Sizonenko, C. Borradori-Tolsa, J.-F. Mangin, P.S. Hüppi, "Mapping the early cortical folding process in the preterm newborn brain," Cerebral Cortex, vol. 18, no. 6, pp. 1444-1454, 2008.

[9] A. Le Troter, D. Rivière, and O. Coulon, "An interactive Sulcal Fundi Editor in BrainVisa," in Proc of the Organization for Human brain mapping conference, 2011.

[10] C. Clouchoux, D. Rivière, J.-F. Mangin, G. Operto, J. Régis, O. Coulon, "Model-driven parameterization of the cortical surface for localization and inter-subject matching.," NeuroImage, vol. 50, no. 2, pp. 552-66, 2010.

[11] J. Lefèvre, D. Germanaud, C. Fischer, R. Toro, D. Riviere, and O. Coulon, "Fast surface-based measurements using first eigenfunction of the Laplace-Beltrami Operator: Interest for sulcal description," in 9th IEEE Int. Symp. on Biomedical Imaging. 2012, pp. 1527-1530. 